\title{
Classification Approach towards Ranking and Sorting Problems
}

\author{
Shyamsundar Rajaram ${ }^{1}$, Ashutosh Garg ${ }^{2}$, Xiang Sean Zhou ${ }^{3}$, and Thomas S. Huang ${ }^{1}$ \\ 1 University of Illinois, Urbana, IL \\ $\{$ rajaram1, t-huang\}@uiuc.edu \\ 2 IBM Almaden Research Center, San Jose, CA \\ ashutosh@us.ibm.com \\ 3 Siemens Corporate Research, Princeton, NJ \\ xzhou@scr.siemens.com
}

\begin{abstract}
Recently, ranking and sorting problems have attracted the attention of researchers in the machine learning community. By ranking, we refer to categorizing examples into one of $K$ categories. On the other hand, sorting refers to coming up with the ordering of the data that agrees with some ground truth preference function. As against standard approaches of treating ranking as a multiclass classification problem, in this paper we argue that ranking/sorting problems can be solved by exploiting the inherent structure present in data. We present efficient formulations that enable the use of standard binary classification algorithms to solve these problems, however the structure is still captured in our formulations. We further show that our approach subsumes the various approaches that were developed in the past. We evaluate our algorithm on both synthetic datasets and for a real world image processing problem. The results obtained demonstrate the superiority of our algorithm over multiclass classification and other similar approaches for ranking/sorting data.
\end{abstract}

\section{Introduction}

Consider the problem of ranking movies. The goal is to predict if a movie fan will like a certain movie or not. One can probably extract a number of features like - actors playing the lead role, setting of the movie (urban or rural), type of movie (romantic or a horror movie etc). Based on this, a set of possible ratings for a movie could be - run-tosee, very-good, good, only-if-you-must, do-not-bother, and avoid-at-all-cost. At another time, given a list of movies one might like to obtain a ordered list where the order in the list represents his preferences or choices. Here we observe that, in both these problems, the labels themselves do not carry much meaning. The information is captured by the relative ranks or order given to the movies. Henceforth, we refer to the first problem as ranking problem and the second problem as sorting problem.

Most of the research in the machine learning community has gone into developing algorithms which are good for either classification or regression. Given that, some of the researchers have attempted to solve the ranking and sorting problems by posing them as either multiclass classification or regression problems. In this paper, we argue that this is not the right approach. If the ranking problem is posed as a classification problem then the inherent structure present in ranked data is not made use of and hence generalization ability of such classifiers is severely limited. On the other hand, posing the task of sorting as a regression problem leads to a highly constrained problem. 
This paper starts with the theoretical analysis relating the problem of sorting with classification. We show that the VC-dimension of a linear classifier is directly related to the rank-dimension (defined later) of a linear classifier. We use the intuition developed by studying the complexity of sorting/ranking problems to show how these can be reduced to a standard classification problem. This reduction enables use of both powerful batch learning algorithms like SVM and online classification algorithms like Winnow and Perceptron. The online version of our algorithm subsumes the previously proposed algorithm Pranking [2] and at the same time is much easier to implement. Further, in many ranking problems, because of the users preference, only few features may be active at any given time and as such Winnow [9] is a better choice than Perceptron. We further extend the results presented in the paper to handle the case when it may not be possible to learn a linear ranker in the original data space. In particular, we make use of kernel methods and show how one can learn a non linear ranker for both the ranking and sorting problems. This formulation is similar in spirit to the work by [4], however in our approach there is a significant reduction in the computational complexity.

At this point, we would like to note that the basic problem formulation presented in this paper is different from the formulation that has been assumed in algorithms like Cranking [7]. The model adopted in the latter is specifically meant to learn the sorting of the data based on the ordering of the data given by other experts. The problem considered in this paper however deals with the case when we actually do not have the orderings from individual experts but instead have some feature based representation of the data. For example, given some documents/images that need to be sorted or ranked, we will extract the features from these documents/images and learn a ranker on top of these. This is the view which has also been adopted in $[2,4]$.

Recently, there has also been work to solve the problem of multiclass classification by considering ordering information in class attributes, thereby posing it as a ranking problem. In [3], Frank et al. transform the data in such a way that the $k$-class ordinal classification problem reduces to $k-1$ binary class problems. Krammer et al. [13] use a modification of S-CART (Structural Classification And Regression Trees) to perform ordinal classification. The algorithm that we propose in this paper, can be easily adapted to solve the problem of multiclass classification if the ordering of the classes are known.

The organization of the paper is as follows. We start off by explaining some notations and give the formal definition of the problem. We will also explain the model adopted to solve the problem. In Sec. 3, we give the relationship between the rankdimension and VC-dimension of a linear classifier. Sec. 4 gives the main result of the paper. In this section, we show how one can reduce the ranking and sorting problems to a classification problem. Based on the results from this section, we show how one can use SVM and other kernel algorithms in Sec. 5. Finally, in Sec. 6 we give results on both synthetic data and test our ranking algorithm on a novel image processing problem.

\section{Notations and Problem Definition}

Consider a training sample of size $m$ say $S=\left\{\left(x_{1}, y_{1}\right),\left(x_{2}, y_{2}\right), \ldots,\left(x_{m}, y_{m}\right)\right\}, x_{i} \in$ $\mathcal{X}, y_{i} \in \mathcal{Y}$ where $\mathcal{X}$ is the domain from which each training example comes and $\mathcal{Y}$ is the space from which labels are assigned to each example. The labels $y_{i}$ are referred to 
as the ranks given to the examples. As mentioned earlier, in this paper we have adopted a feature based representation of the data and we assume that $\mathcal{X}$ is $n$ dimensional space of reals $\Re^{n}$. Under this, for any $x_{i}, x_{j} \in \mathcal{X}$ we have $x_{i}-x_{j} \in \mathcal{X}$.

For ranking problem, $\mathcal{Y}=\{1, \ldots, K\}$ where $K$ is the maximum rank that can be taken by any example. This is similar to the multiclass classification problem. However the spirit of the ranking problem is very different. The ranks relate to the degree of interest/confidence a person has in that instance. Given an example with rank $k$, all the examples with rank less than $k$ are less interesting and all the examples with rank more than $k$ are more interesting. Such a relationship/viewpoint does not exist in case of multi-class classification problem. In general we will assume that $K$ (the maximum rank) is fixed for a given problem and will treat the case when $K$ is same as the size of the dataset separately. We refer to this latter case as the sorting problem. In the sorting problem, each training example has a distinct rank (this rank will be referred to as the order in the dataset) which refers to the relative position of that instance in the dataset. In this case, one cannot treat it as a multiclass problem as the number of classes depend on the size of the data and the interest is in learning a function that can give relative ordering instead of absolute class labels.

\subsection{The Ranking Model}

In this paper, we adopt a functional approach to solve the ranking problem. Given a data point $x$ or a set of data points $S$, we learn a ranker $f: \mathcal{X} \rightarrow \mathcal{Y}$. Depending upon whether $K$ is fixed or it is $m$, our choice of function $f$ will be different. In general, when $K<m$ is fixed, we will refer to it as the ranking problem where as when $K=m$, we will refer to it as the sorting problem.

In this paper, we assume that there exists an axis in some space such that when data is projected on to this axis, the relative position of the data points captures the model of user preferences. In the case of the sorting problem, we will treat $f$ to be a linear function whose value is the signed distance from some hyperplane $h$. That is $f\left(x_{i}\right)=h^{T} x_{i}$. The information about the relative order of the datapoints will be captured by the distance from the hyperplane. During the testing phase, given a set of datapoints, the sorted set of datapoints with respect to their order will be obtained by sorting them with respect to the raw value $f(x)$.

We will adopt a similar framework to solve the ranking problem. However, in this case, in addition to learning $h$ (as in the previous case), we also learn a number of thresholds $(K-1)$ corresponding to the different ranks that will be assigned to data. The learned classifier in this case will be expressed as $\left(h, \theta_{1}, \ldots, \theta_{K-1}\right)$ with the thresholds satisfying $\theta_{1}<\theta_{2} \ldots<\theta_{K-1}$. The ranking rule in this case is

$$
f(x)= \begin{cases}1 & \text { if } h^{T} x<\theta_{1}, \\ i & \text { if } \theta_{i-1}<h^{T} x<\theta_{i}, \quad i \neq 1, K \\ K & \text { if } \theta_{K-1}<h^{T} x\end{cases}
$$

Although, this model of ranking may seem too simplistic, as we show in Sec. 3 it is quiet powerful and we give some analysis relating VC-dimension of the learned classifier to what we call rank-dimension of the data. Later in Sec. 5, we will show how 
one can extend the above framework to the case where learning needs to be done in a space different from the original space. In such a case, learning is done in some high dimensional space by making use of kernels for the mapping.

\section{Complexity of Ranking vs Classification}

It has been argued [1] that the ranking problem is much harder than the classification problem. Although this is true in the particular view adopted by [1], in this paper we present an alternate viewpoint. We analyze the complexity of the ranking problem from the view of the VC-dimension. We define the variant of the VC-dimension, called rankdimension, for the ranking problem as follows: if the data points are ranked with respect to the value of the functional evaluated on a particular data point, then we say that the rank dimension of the functional is the maximum number of points that can be ranked in any arbitrary way using this functional.

Theorem 1. The rank dimension of a linear functional is same as its VC-dimension. Following the notation given in Sec. 2, it holds for all $x_{i}, x_{j} \in \mathcal{X}$ we also have $x_{i}-x_{j} \in$ $\mathcal{X}$ and $x_{j}-x_{i} \in \mathcal{X}$

Proof. Let us consider the case of linear classifier $h \in \Re^{n}$. Say one observes a set of $m$ points $S=\left\{x_{1}, x_{2}, \ldots, x_{m}\right\}$, with the corresponding ranks $y_{1}, y_{2}, \ldots, y_{m}$.

Clearly if we can rank a set of $m$ points in any arbitrary way using a functional, then we can always shatter them (at the cost of one additional dimension corresponding to the threshold). Consider a subset $S_{0} \subset S$ such that we want to label all the points that belong to $S_{0}$ as negative and all the points that belong to $S$ but not to $S_{0}$ as positive (i.e. $\left.S \backslash S_{0}\right)$. Now, if we rank all the points in such a way so that the rank of all the points in $S_{0}$ is less than the rank of all the points in $S \backslash S_{0}$ then we can do the classification by just thresholding based on the rank. This shows that the rank-dimension of any functional cannot be more than the "VC-dimension" of the same functional.

We know that the VC-dimension of a linear classifier in $n$ dimensional space is $n+1$. That is any set of $n+1$ points (assuming general positions) in $n$ dimensional space can be shattered by a $n$ dimensional linear classifier. Now we show that any set of $n+1$ points can be ranked in any arbitrary way using a linear classifier in $n$ dimensional space. Given any arbitrary ranking of the points, lets re-label the points such that $\operatorname{rank}\left(x_{1}\right)<\operatorname{rank}\left(x_{2}\right)<\ldots<\operatorname{rank}\left(x_{n+1}\right)$. Define a new set of points $S^{\prime}=$ $\left\{0, x_{2}-x_{1}, x_{3}-x_{2}, \ldots, x_{n+1}-x_{n}\right\}$. Now, if we label the points as $\{-1,1,1, \ldots, 1\}$. The cardinality of set $S^{\prime}$ is $n+1$ (n-difference vectors and one 0 vector.) Also it is easy to see that all points in $S^{\prime}$ lie in $\Re^{n}$. Now, from the VC-dimension theory, we know that there exists a linear classifier in $n$ dimensional space that can shatter $S^{\prime}$ according to the labelling given above. Let this linear classifier be $h$, with classification as $\operatorname{sign}\left(h^{T} x\right)$. Then for correct classification $h^{T}\left(x_{i}-x_{i-1}\right)>0 \Rightarrow h^{T} x_{i}>h^{T} x_{i-1}$. That is the distance of the original points from the hyperplane does corresponds to the specified ranking. Hence, we have shown that any pair of $n+1$ points can be ranked in any arbitrary fashion by a $n$ dimensional classifier and at the same time we have also shown that the rank dimension cannot be more than the VC-dimension. This shows that the rank-dimension of any classifier is same as its VC-dimension. 
This is a very interesting result as it shows that the complexity of the hypothesis space for the two problems is same. However, as of now, we are not clear about the relation between the growth function for the two problems. Further, the relation between the computational complexity of the two problems has to be studied.

\section{Ranking, Sorting and Classification}

In the previous section, we saw the close relationship between the classification and the sorting problem. It is clear that if we can solve a sorting problem then we can also solve a ranking problem (by giving any arbitrary order to all the data points within the same rank class). However, it turns out that computationally both problems demand individual treatment. In this section, we present two approaches to solve this problem. The first is referred to as the difference space approach while the second is referred to as the embedded space approach.

\subsection{Difference Space Approach}

Given a training set $S$, define a new set $S_{d}$ of difference vectors $x_{i j}^{d}=x_{i}-x_{j} ; \forall i, j$ : $y_{i} \neq y_{j}$ and their corresponding labels $y_{i j}^{d}=\operatorname{sign}\left(y_{i}-y_{j}\right)$. This leads to a dataset ${ }^{1}$ of size $O\left(\mathrm{~m}^{2}\right)$. Learning a linear classifier for this problem would be same as learning a ranker $h$. Once such a ranker is learned, the thresholds for the ranking problem can easily be computed. This formulation is same as the one proposed by [4]. Computational complexity of most of the learning algorithms depend on the size of the training data and a quadratic increase in the size of the data will certainly make most of the existing algorithms impractical. Hence, we propose to generate difference vectors only among the adjacent rank classes. Formally, given a training set $S$, obtain a new set $S_{d}$ made up of difference vectors $x_{i j}^{d}=x_{i}-x_{j} ; \forall i, j: y_{i}=y_{j}+1$ and their corresponding labels $y_{i j}^{d}=+1$. This would result in a dataset with only positive examples. Again, most standard classification algorithms behave well if the number of positive examples is close to the number of negative examples. To get around this problem, once such a dataset is obtained, multiply each example $x_{i j}^{d}$ and the corresponding label $y_{i j}^{d}$ by $q_{i j}$ where $q_{i j}$ is a random variable taking values $\{-1,1\}$ with equal probabilities. Clearly, learning a linear classifier over this dataset will give a ranker $h$ which will be same as the one obtained in the previous case. The size of the dataset in this case is $O\left(\frac{m^{2}}{K}\right)$. For a small $K$ (which is the case in most $K$-ranking problems) this is still too large to handle. However, interestingly, for the sorting problem, the size of the new dataset is of the same order as the size of the old dataset and therefore this problem can be solved easily. Next, we present an approach that specifically handles the ranking problem without exploding the size of the training dataset.

\subsection{Embedded Space Approach}

In this section, we present a new framework to handle the ranking problem which can be seen as the main contribution of this paper. It is a novel formulation that allows one

\footnotetext{
${ }^{1}$ The different elements of this dataset are not independent of one another.
} 
to map a ranking problem to a standard classification problem without increasing the size of the dataset. The embedded space approach presented in this section is similar in spirit to the model presented in [12], however as we will see shortly in our model, the dimension of the new space does not grow linearly as the one presented in their paper. Fig. 1 graphically depicts the ranking framework. The distance from the hyperplane $h$ of a datapoint $x$ is mapped to a one dimensional space. In this space, $\theta_{1}, . ., \theta_{K-1}$ are the different thresholds against which the distance is compared. Note that $h^{T} x_{j} ; \forall x_{j}$ having rank $i$ results in a range represented by its left end point $\theta_{i-1}$ and its right end point $\theta_{i}$. Define $\alpha_{i}=\theta_{i+1}-\theta_{i} ; 1 \leq i \leq K-1$. For the data items belonging to rank 1 , there is no lower bound and for all the data items belonging to rank $K$ there is no upper bound. By construction, it is easy to see that $\alpha_{i}>0 ; \forall i$. Note that data point $x_{j}$ having rank $i>1$ will satisfy, (assuming $\alpha_{0}=0$.)

$$
h^{T} x_{j}>\theta_{i-1} ; \quad h^{T} x_{j}+\alpha_{i-1}>\theta_{i} ; \quad h^{T} x_{j}+\sum_{k=i-1}^{K-2} \alpha_{k}>\theta_{K-1}
$$

Similarly, for an example with rank $i<K$, (assuming $\theta_{K}=\infty, \alpha_{K-1}=0$ )

$$
h^{T} x_{j}<\theta_{i} ; h^{T} x_{j}+\alpha_{i}<\theta_{i+1} ; h^{T} x_{j}+\sum_{k=i}^{K-2} \alpha_{k}<\theta_{K-1}
$$

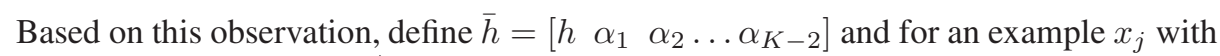
rank $1<i<K$, define $\bar{x}_{j}^{+}, \bar{x}_{j}^{-}$as $n+K-2$ dimensional vectors with

$$
\bar{x}_{j}^{+}[l]=\left\{\begin{array}{ll}
x_{j}[l] & 1 \leq l \leq n, \\
0 & n<l<n+i-1, \\
1 & n+i-1 \leq l \leq n+K-2 .
\end{array} \bar{x}_{j}^{-}[l]= \begin{cases}x_{j}[l] & 1 \leq l \leq n \\
0 & n<l<n+i \\
1 & n+i \leq l \leq n+K-2\end{cases}\right.
$$

For an example $x_{j}$ with rank $i=1$, we define only $\bar{x}_{j}^{-}$as above and for an example with rank $i=K$, we define only $\bar{x}_{j}^{+}$again as above. This formulation assumes that $\theta_{K-1}=$ 0 . It is easy to see that one can assume this without loss of generality (by increasing the dimension of $x$ by 1 one can get around this.) Once we have defined $\bar{x}_{j}^{+}, \bar{x}_{j}^{-}$, the ranking problem simply reduces to learning a classifier $\bar{h}$ in $n+K-2$ dimensional space such that $\bar{h}^{T} \bar{x}_{j}^{+}>0$; and $\bar{h}^{T} \bar{x}_{j}^{-}<0$. This is a standard classification problem with at most $2 m$ training examples, half of which have label +1 (examples $\bar{x}_{j}^{+}$) and rest have label -1 (examples $\bar{x}_{j}^{-}$). Even though, the overall dimension of the data points and the weight vector $h$ is increased by $K-2$, this representation limits the number of training data points to be $O(m)$. Note that although, we have slightly increased the

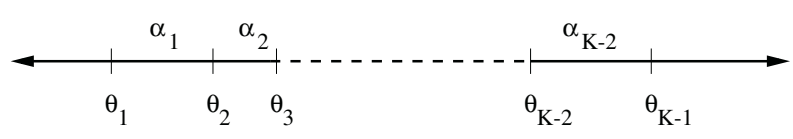

Fig. 1. Graphical representation of the ranking framework. 
dimension (by $K-2$ ), the number of parameters that needs to be learned is still the same (the classifier and the thresholds). Interestingly, any linear classification method can now be used to solve this problem. It is easy to prove that if there exists a classifier that learns the above rule with no error on the training data then all the $\alpha_{i}$ 's are always positive which is a requirement for the classification problem to be same as ranking problem. Next we show how one can use kernel classifiers (SVM) to solve the ranking problem for datasets where there may not exist a linear ranker.

\section{Kernel Classifiers - SVM}

In many real world problems it may not be possible to come up with a linear function that would be powerful enough to learn the ranking of the data. In such a scenario, standard practice is to make use of kernels which allow nonlinear mapping of data. We will denote a kernel as $\mathcal{K}(\cdot, \cdot)=\phi^{T}(\cdot) \phi(\cdot)$ which corresponds to using the non-linear mapping $\phi(\cdot)$ over the original feature vector.

Solving Sorting Problems. For the sorting problem, we propose to learn a linear classifier over the difference feature vectors and then use this learned classifier to sort the data. While using non linear mapping $\phi$ with corresponding kernel $\mathcal{K}$, this would imply

$$
\text { Learn } h: y_{i j}^{d}\left(\phi(h)^{T} \phi\left(x_{i j}^{d}\right)\right)>0 \Rightarrow h: y_{i j}^{d}\left(\phi(h)^{T} \phi\left(x_{i}-x_{j}\right)\right)>0
$$

However, we note that $\phi\left(x_{i}-x_{j}\right) \neq \phi\left(x_{i}\right)-\phi\left(x_{j}\right)$ because $\phi()$ is a non linear mapping. To get around this, we adopt a different strategy (also proposed in [4]). Instead of solving the classification problem over the difference vector in the original space, we solve the classification problem over the difference vectors in the projected space.

$$
\text { Learn } h: y_{i j}^{d}\left(\phi(h)^{T}\left(\phi\left(x_{i}\right)-\phi\left(x_{j}\right)\right)>0\right.
$$

Interestingly, it can be solved easily by defining a new kernel function(It can be easily verified that this is a Kernel function) as

$$
\mathcal{K}\left(x_{i}-x_{j}, x_{l}-x_{m}\right)=\mathcal{K}\left(x_{i}, x_{l}\right)+\mathcal{K}\left(x_{j}, x_{m}\right)-\mathcal{K}\left(x_{i}, x_{m}\right)-\mathcal{K}\left(x_{j}, x_{l}\right)
$$

Solving Ranking Problems. For solving the ranking problem, we have proposed the mapping given in Eqn. 4. One has to be careful in using kernel classifiers with this mapping. To see this, note that if $x_{j}$ has rank $i$, then $\bar{h}^{T} \bar{x}_{j}^{+}>0 \Rightarrow h^{T} x_{j}>\theta_{i-1} ; \bar{h}^{T} \bar{x}_{j}^{-}<$ $0 \Rightarrow h^{T} x_{j}>\theta_{i}$ but $\mathcal{K}\left(\bar{h}, \bar{x}_{j}^{+}\right)=\phi(\bar{h})^{T} \phi\left(\bar{x}_{j}^{+}\right)>0 \nRightarrow \phi(h)^{T} \phi\left(x_{j}\right)>\theta_{i-1}$ This is again because of the nonlinearity of the mapping $\phi()$. However, one can again get around this problem by defining a new kernel function. For a kernel function $\mathcal{K}$ and the corresponding mapping $\phi$, lets define a new kernel function $\overline{\mathcal{K}}$ and with the corresponding mapping $\phi()$ as

$$
\phi(\bar{x})=[\phi(x), \bar{x}[n+1: n+K-2]] ; \quad \bar{\phi}(\bar{h})=[\phi(h), \bar{h}[n+1: n+K-2]]
$$

Note that, only the first $n$ dimensions of $\bar{x}$ corresponding to $x$ are projected to a higher dimensional space. The new kernel function can hence be decomposed into sum of two kernel functions where the first term is obtained by evaluation of kernel over the first $n$ 
dimensions of the vector and second term is obtained by evaluating a linear kernel over the remaining dimensions.

$$
\overline{\mathcal{K}}\left(\bar{x}_{i}, \bar{x}_{j}\right)=\mathcal{K}\left(x_{i}, x_{j}\right)+\bar{x}_{i}[n+1: n+K-2]^{T} \bar{x}_{j}[n+1: n+K-2]
$$

However, when using the SVM algorithm with kernels one has to be careful as when working in the embedded space, learning algorithms minimize the norm of $\bar{h}$ and not $h$ as should have been the case. In the next section, we introduce the problem of ordinal regression and show how one can get around this problem.

\subsection{Reduction to Ordinal Regression}

In this section, we show how one can actually get around the problem of minimizing $\|h\|$ as against minimizing $\|\bar{h}\|$. We want to solve the following problem

$$
\text { minimize } \frac{1}{2}\|h\|^{2} ; \quad \text { subject to } \bar{y}_{j}^{+/-}\left(\bar{h}^{T} \bar{x}_{j}^{+/-}+b\right)>0
$$

Based on the analysis in Sec. 4 , the inequality in the above formulation for $x_{j}$ with rank $y_{j}$ can be written as,

$$
-b-\sum_{l=n+1}^{n+k-2} \bar{h}(l) \bar{x}_{j}^{-}(l)=\theta_{y_{j}}>h^{T} x_{j}>-b-\sum_{l=n+1}^{n+k-2} \bar{h}(l) \bar{x}_{j}^{+}(l)=\theta_{y_{j}-1} ; \quad 1<i \leq K
$$

In this analysis, we will assume that with respect to threshold $\theta_{i}$ 's, there is a margin of at least $\epsilon$ such that for any datapoint $x_{j}$ with corresponding rank $y_{j}$, we have

$$
\theta_{y_{j}-1}+\epsilon<h^{T} x_{j} ; \quad 1<y_{j} \leq K
$$

Now, the problem given in Eqn. 10 can be reframed as

$$
\text { minimize } \frac{1}{2}\|h\|^{2} ; \quad \text { subject to } h^{T} x_{j}<\theta_{y_{j}} ; \forall 1 \leq y_{j}<K h^{T} x_{j}>\theta_{y_{j}-1}+\epsilon ; 1<y_{j} \leq K
$$

This leads to the following lagrange formulation,

$$
L_{P}=\frac{1}{2}\|h\|^{2}+\sum_{j=1}^{m-m_{K}} \gamma_{j}^{+}\left(h^{T} x_{j}-\theta_{y_{j}}\right)+\sum_{j=m_{1}+1}^{m} \gamma_{j}^{-}\left(\theta_{y_{j}-1}+\epsilon-h^{T} x_{j}\right)-\sum_{j} \gamma_{j}^{+}-\sum_{j} \gamma_{j}^{-}
$$

where $m_{i}$ refers to number of elements having rank ' $i$ '. The ranker $h$ is obtained by minimizing the above cost function under the positivity constraints for $\gamma_{j}^{+}$and $\gamma_{j}^{-}$. Dual formulation $L_{d}$ of the above problem can be obtained by following steps as in [11],

$$
L_{d}=-\frac{1}{2} \sum_{i=1}^{n} \sum_{j=1}^{n}\left(\gamma_{i}^{-}-\gamma_{i}^{+}\right)\left(\gamma_{j}^{-}-\gamma_{j}^{+}\right) \mathcal{K}\left(x_{i}, x_{j}\right)-\sum_{j} \gamma_{j}^{+}-\sum_{j} \gamma_{j}^{-}
$$

with constraints,

$$
\sum_{p=m_{i-1}+1}^{m_{i}} \gamma_{p}^{+}=\sum_{p=m_{i}+1}^{m_{i+1}} \gamma_{p}^{-} ; \quad \forall i \in[2, K-1]
$$


Table 1. (left)Comparison of sorting algorithms for linear data. (right) Comparison of sorting algorithms for nonlinear data.

\begin{tabular}{|l|c|}
\hline Algorithm & Ave. No. of Transpositions \\
\hline Perceptron & 36.31 \\
\hline Winnow & 35.68 \\
\hline linear-SVM & $\mathbf{3 0 . 9 2}$ \\
\hline
\end{tabular}

\begin{tabular}{|l|c|}
\hline Algorithm & Ave. No. of Transpositions \\
\hline Perceptron & 62771 \\
\hline Winnow & 62840 \\
\hline kernel-SVM & $\mathbf{1 4 5 6 . 4}$ \\
\hline
\end{tabular}

We have introduced $\gamma_{l}^{+}, \gamma_{m}^{-} \forall l \in\left[n_{K-1}+1, n_{K}\right]$ and $m \in\left[1, n_{1}\right]$ for simplicity of notation. These are deterministic quantities with value 0 . It is interesting to note that, Eqn. 11 has the same form as a regression problem. The value of $\theta_{i}$ 's is obtained using Kuhn Tucker conditions.

\section{Experiments}

In this section, we present experimental results of the various algorithms that have been outlined in this paper. We start off by giving results obtained on synthetic data and then we show results of the performance of ranking for a novel image processing application.

\subsection{Synthetic Data}

Sorting. First, we compare the performance of Perceptron, Winnow and SVM for sorting problem on linear data. Linear data is obtained by generating $N$ dimensional data $x_{i} ; 1 \leq i \leq m$. Data points are sorted based on the value of inner product $w^{T} x_{i}$ where $w$ is a randomly generated $N$ dimensional vector. In the following experiments we choose $N=5, M=700$ with 200 examples as training data and rest as test data. The performance of the three algorithms was analyzed by computing the minimum number of adjacent transpositions [7] needed to bring the sorting produced by the learned ranker $\hat{Y}$ to the ground truth $Y$.

In Table. 1, we show the averaged number of transpositions normalized by the length of the sequence for the three methods. It can be observed that linear-SVMs are slightly better than the online learning rules.

Next we compare the performance of the three algorithms on nonlinear data. The data was generated as above with the addition of quadratic non-linearity in the data when obtaining the ground truth sorting. Table. 1 shows the performance of the three algorithms. A radial basis function is used as kernel and it is clear from the results that kernel-SVMs handle non linear data very well. Fig. 2 shows an instance of sorting performed by kernel SVMs for nonlinear data. In this figure, the data is plotted with respect to the ground truth sorting. It is clearly evident that the non-linear SVM is able to capture the inherent nonlinearity.

Ranking. In addition to various ranking algorithms proposed in the paper, one can treat ranking as a multiclass classification problem. We present a comparison of various ranking algorithms on synthetic data and study how their behavior varies with the increase in number of classes/ranks. In particular, we analyze ranking algorithms namely 


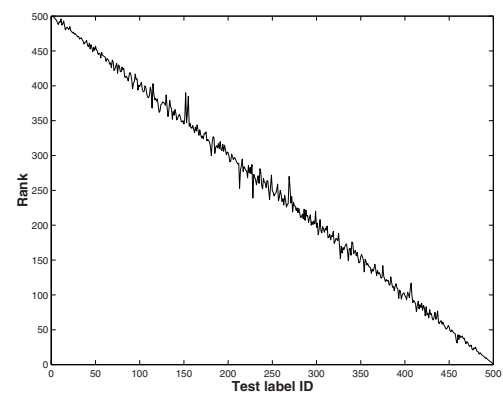

Fig. 2. An example of sorting done using kernel-SVM for nonlinear data. The ideal ranking is a straight line.

Table 2. (left)Comparison of Ranking algorithms for linear data. The rows represent the values of $K$ and the columns correspond to the algorithms A1:SVM using ordinal regression, A2:multiclass SVM, A3:SVMs using appending, A4:Perceptron, A5:Winnow. (right) Comparison of Kranking algorithms for nonlinear data. The rows represent different $K$ values ranging from 3 to 10 and the columns correspond to the algorithms A1:SVM using ordinal regression, A2:multi-class SVM, A3:SVMs using appending, A4:Perceptron, A5:Winnow.

\begin{tabular}{|l|c|c|c|c|c|}
\hline & A1 & A2 & A3 & A4 & A5 \\
\hline $\mathrm{K}=3$ & 7.8 & 15.6 & $\mathbf{7 . 2}$ & 10.1 & 9.1 \\
\hline $\mathrm{K}=4$ & $\mathbf{8 . 3}$ & 34.7 & $\mathbf{8 . 3}$ & 7.0 & 6.2 \\
\hline $\mathrm{K}=5$ & $\mathbf{4 . 5}$ & 52.2 & $\mathbf{4 . 5}$ & 9.0 & 5.6 \\
\hline $\mathrm{K}=6$ & $\mathbf{5 . 1}$ & 68.2 & 5.9 & 10.6 & 6.0 \\
\hline $\mathrm{K}=7$ & $\mathbf{9 . 8}$ & 72.5 & $\mathbf{9 . 8}$ & 8.3 & 8.5 \\
\hline $\mathrm{K}=8$ & $\mathbf{7 . 7}$ & 82.0 & $\mathbf{7 . 7}$ & 8.9 & 8.1 \\
\hline $\mathrm{K}=9$ & 8.5 & 83.6 & $\mathbf{8 . 2}$ & 11.1 & 9.1 \\
\hline $\mathrm{K}=10$ & 8.0 & 93.3 & $\mathbf{7 . 8}$ & 9.6 & 6.2 \\
\hline
\end{tabular}

\begin{tabular}{|l|c|c|c|c|c|}
\hline & A1 & A2 & A3 & A4 & A5 \\
\hline $\mathrm{K}=3$ & $\mathbf{2 2 . 9}$ & 51.9 & 24.3 & 78.6 & 83.2 \\
\hline $\mathrm{K}=4$ & 29.3 & 65.5 & $\mathbf{2 9 . 0}$ & 95.1 & 97.0 \\
\hline $\mathrm{K}=5$ & 35.1 & 87.4 & $\mathbf{3 4 . 8}$ & 105.5 & 105.5 \\
\hline $\mathrm{K}=6$ & $\mathbf{3 7 . 6}$ & 93.6 & 37.7 & 113.6 & 108.2 \\
\hline $\mathrm{K}=7$ & 36.1 & 104.3 & $\mathbf{3 5 . 6}$ & 114.4 & 116.1 \\
\hline $\mathrm{K}=8$ & $\mathbf{3 0 . 9}$ & 107.5 & 31.1 & 116.0 & 111.8 \\
\hline $\mathrm{K}=9$ & $\mathbf{4 0 . 7}$ & 108.6 & 41.1 & 116.8 & 117.1 \\
\hline $\mathrm{K}=10$ & 38.3 & 118.0 & $\mathbf{3 8 . 2}$ & 123.2 & 130.3 \\
\hline
\end{tabular}

Perceptron, Winnow, SVM using ordinal regression, multi-class SVM and SVMs using appending (by mapping the data to new embedded space as explained in Sec. 4. Multi-class SVM learns $K$ classifiers where each classifier distinguishes one class corresponding to particular rank from the rest of the classes. SVM using ordinal regression and SVM using appending are implementation of the theory discussed in Sec. 5.1 and Section. 4 respectively. Linear data is generated and sorted as before. Now the sorted set is divided into $K$ equally sized groups and labels are assigned to them ranging from $[1, K]$ such that, any item in group $i$ must be placed lower than any other element in group $i-1$ with respect to some linear hyperplane (unknown to the algorithm). The results are tabulated in Table. 2.

Next, we give the results for the case when there is no linear ranker. Nonlinear data is again generated as discussed earlier Table. 2 shows the results obtained in tabular format. It is clear from both Table. 2 that SVMs using ordinal regression and appending do significantly better than the multi-class SVMs because the former methods capture the inherent structure in the data. 


\subsection{Automatic Image Focusing}

A very nice application of ranking datasets is to rank images $I$ capturing a particular scene based on the level of focus and hence perform defocusing by picking the image which has the highest rank among all the images. We choose this particular application [6] [10][5] to demonstrate the applicability of ranking formulation presented in the paper towards solving the task of automatically extracting the best focused image.

To study the image focusing problem, we started by experimenting with synthetic data. 50 images were take from the corel database. As has been argued in literature, Gaussian blurring is closely related to the noise in a badly focused image and hence we used this method to obtain images which are not focused properly. In particular, each image is blurred two times giving us three images with varying level of focus. The more the blurring is, the worse is the appearance of the image. This whole process resulted in 150 images with varying level of focus. Each of these images were resized to obtain a 120x80 image resulting in a 9600 dimensional vector. Instead of representing the image in such crude form, we use Gabor 2D wavelets [8] to represent each of the image by a 64 dimensional vector. The ranking algorithm discussed earlier is used with $\operatorname{rbf}$ (radial basis function) kernel to learn the ranking function. The learned function is then evaluated on the remaining 120 images corresponding to 40 different scenes (three images correspond to each scene). For each scene, the testing task is to figure out the clearest (best focused) image out of the three images. In $95 \%$ of the cases (that is in all but two cases), the original image (without Gaussian noise) is picked as the best focused image.

The real test of the algorithm is when it is subject to real data. To do this, we used a digital camera (IBM Web camera) and captured 42 scenes from it. For each scene, three images were taken by manually distorting the focus. Some of these images are shown in Fig. 3. Note that the difference between the focused and unfocused image is very minor (this makes the problem very hard). To test it, we used a novel approach in which we took small amount of real data (since we want to test it on as much real data as possible) -5 scenes and used all the synthetic data as the training set. Again all the images were of size - 120x80 resulting in a 9600 dimensional feature vector. As with synthetic data, the Gabor signatures are used to represent these image as a 64 dimensional vector. Again, we made use of our ranking algorithm with rbf kernel function to learn the ranker. When tested on remaining 37 scenes, we found that except for 2 cases, in 35 cases the algorithm picked the correct image. Some of the images ranked by our system are presented in Fig. 3. The strength and generalization capabilities of the algorithm are clearly evident from the fact that the classifier which was learned with only a small number of real training images (and synthetic images) did so well on the test data.

\section{Summary}

In this paper, we have presented an algorithm to reduce both sorting and ranking problems to a binary classification problem. We have also shown how one can make use of kernels to solve these problems in higher dimensional spaces. Moreover, our reduction of the ranking problem to a binary classification problem results in a straightforward 

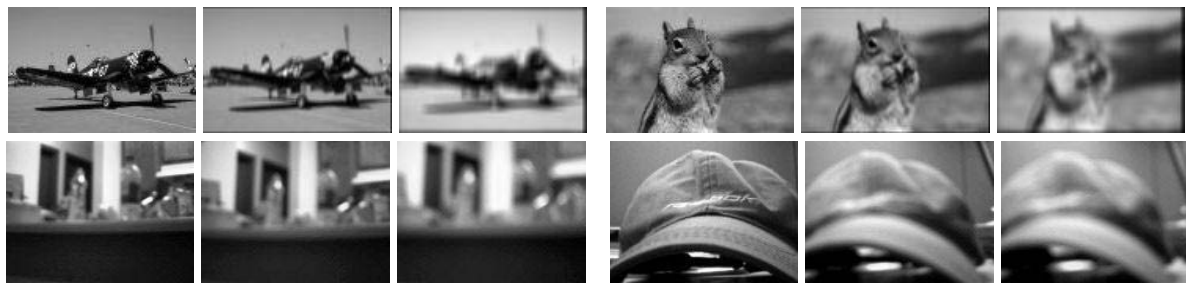

Fig. 3. Some synthetic(top row) and real(bottom row) images used to test our system.

application of online/batch classification algorithms. The main contribution of the paper is that training can be done with linear amount of data using a novel formulation of the ranking problem. Our results on synthetic and real data shows that these algorithms are viable and can be used in practice.

\section{References}

1. W. Cohen, R. Schapire, and Y. Singer. Learning to order things. Artificial Intelligence Research, 10:243-279, 1999.

2. K. Crammer and Y. Singer. Pranking with ranking. In T. G. Dietterich, S. Becker, and Z. Ghahramani, editors, Advances in Neural Information Processing Systems 14, Cambridge, MA, 2002. MIT Press.

3. E. Frank and M. Hall. A simple approach to ordinal classification. Lecture Notes in Computer Science, 2167:145-??, 2001.

4. R. Herbrich, T. Graepel, and K. Obermayer. Large margin rank boundaries for ordinal regression. Advances in Large Margin Classifiers, MIT press, pages 115-132, 2000.

5. B. K. P. Horn. Focusing. Technical Report AIM-160, Massachusetts Institute of Technology, May 1968.

6. R. A. Jarvis. Focus optimisation criteria for computer image processing. Microscope, 24(2):163-180, 1976.

7. G. Lebanon and J. Lafferty. Cranking: Combining rankings using conditional probability models on permutations. In Proceedings of the 19th International Conference on Machine Learning (ICML), San Francisco, CA, 2002. Morgan Kaufmann Publishers.

8. T. S. Lee. Image representation using $2 \mathrm{~d}$ gabor wavelets. IEEE Transactions on Pattern Analysis and Machine Intelligence, 18(10):959-971, 1996.

9. N. Littlestone. Learning quickly when irrelevant attributes abound: A new linear threshold algorithm. Machine Learning, 2(4):115-132, 1988.

10. J. F. Schlag, A. C. Sanderson C. P. Neumann, and F. C. Wimberly. Implementation of automatic focusing algorithms for a computer vision system with camera control. Technical Report CMU-RI-TR-83-14, Carnegie Mellon University, August 1983.

11. A. Smola and B. Schlkopf. A tutorial on support vector regression. In NeuroCOLT2 Technical Report NC2-TR-1998-030, 1998.

12. D. Roth S. Har-Paled and D. Zimak. Constraint classification: A new approach to multiclass classification. In 13th Interntional Conference on Algorithmic Learning Theory, pages 365379, 2002.

13. B. Pfahringer S. Kramer, G. Widmer and M. de Groeve. Prediction of ordinal classes using regression trees. In International Syposium on Methodologies for Intelligent Systems, pages 426-434, 2000. 